\title{
Cryostat for the provision of liquid hydrogen with a variable ortho-para ratio for a low-dimensional cold neutron moderator
}

\author{
Sebastian Eisenhut ${ }^{1, *}$, Marcel Klaus ${ }^{1}$, Johannes Baggemann ${ }^{2}$, Ulrich Rücker ${ }^{2}$, Yannick Beßler ${ }^{3}$, Alexander \\ Schwab $^{1}$, Christoph Haberstroh ${ }^{1}$, Tobias Cronert ${ }^{2}$, Thomas Gutberlet ${ }^{2}$, Thomas Brückel ${ }^{2}$, and Carsten Lange ${ }^{4}$ \\ ${ }^{1}$ TU Dresden (Bitzer-Chair of Refrigeration, Cryogenics and Compressor Technology), Dresden, Germany \\ ${ }^{2}$ Forschungszentrum Jülich (JCNS), Jülich, Germany \\ ${ }^{3}$ Forschungszentrum Jülich (ZEA-1), Jülich, Germany \\ ${ }^{4}$ TU Dresden (Chair of Hydrogen and Nuclear Energy), Dresden, Germany
}

\begin{abstract}
A significant contribution to the enhancement of the neutron brilliance achievable with Compact Accelerator-driven Neutron Sources (CANS) can be made by an optimized cold moderator design. When using liquid para- $\mathrm{H}_{2}$ as the moderating medium, the concept of low-dimensional cold moderators can be employed to increase the neutron brightness (as currently foreseen at the European Spallation Source ESS). Para- $\mathrm{H}_{2}$ shows a drop in the scattering cross section by about one order of magnitude around $15 \mathrm{meV}$, resulting in a large deviation between the mean free paths of thermal and cold neutrons. Taking advantage of this effect, the cold moderator geometry can be optimized to allow the intake of thermal neutrons through a relatively large envelope surface and then extracting them in an efficient way towards the neutron guides. One drawback of this solution is the lack of thermalization of the cold neutrons. In the context of the HBS (High Brilliance Neutron Source) project, efforts are made to overcome this problem by increasing the scattering cross section of the $\mathrm{H}_{2}$ in a defined way. The idea is to admix small amounts of ortho- $\mathrm{H}_{2}$, which maintains its large scattering cross section in the region below $15 \mathrm{meV}$. Like this, the neutron spectrum can be shifted towards lower energies and adjusted for the needs of the respective instruments. In a cooperation between TU Dresden and FZ Jülich, an experimental setup has been created to prove the feasibility of this concept. The main component of the experimental setup is a LHe-cooled flow cryostat that enables the separate condensation of a para- $\mathrm{H}_{2}$ and a normal- $\mathrm{H}_{2}$ flow and a subsequent mixing of the two in precise proportions. The resulting $\mathrm{LH}_{2}$ mixture at 17 - $20 \mathrm{~K}$ is fed into a small cold moderator vessel (approx. $200 \mathrm{ml}$ ). In this work, the current status of the setup is presented. The construction and commissioning of the mixing cryostat have been completed and first test runs show that different ortho-para- $\mathrm{H}_{2}$ mixtures can be produced. In the near future, the system will be ready for measurements at a neutron source.
\end{abstract}

\section{Introduction}

The progressive shutdown of existing research reactors in Europe causes an increasing shortage of neutron availability for scattering experiments. To overcome this problem, currently there are efforts being made to establish a network of Compact Accelerator-driven Neutron Sources (CANS). CANS represent a costeffective and versatile way to ensure the neutron provision and establish local facilities with easy access. The Jülich Centre for Neutron Science (JCNS) is currently developing a future CANS, the High Brilliance neutron Source (HBS), in which the whole chain from neutron production to detection is to be optimized according to the experimental needs. The design of the cryogenic moderator is of great importance for an optimized cold neutron provision [1].
Several materials, among them mesitylene, methane, and hydrogen, are currently being investigated as possible candidates for the cryogenic moderators used at HBS. Liquid para- $\mathrm{H}_{2}$ has been used in cold sources since many years, but recently there has been a new trend in para- $\mathrm{H}_{2}$ moderator design: Thanks to the drop in the scattering cross section of para- $\mathrm{H}_{2}$ at a neutron energy around $15 \mathrm{meV}$ (see Figure 1), it is possible to extract neutrons from the cryogenic moderator in a very efficient way due to low self-absorption, if the moderator is implemented as a so-called low-dimensional cold moderator [2].

On the other hand, a major drawback of the extremely low scattering cross section in the cold neutron range is that a good neutron thermalization cannot be reached inside a low-dimensional moderator [1]. As slower neutron spectra are preferred for certain scattering experiments, there is a great interest in further reducing the neutron temperature.

\footnotetext{
${ }^{*}$ Corresponding author: sebastian.eisenhut@tu-dresden.de
} 
As shown in Figure 1, ortho- $\mathrm{H}_{2}$ does, in contrast to para- $\mathrm{H}_{2}$, not exhibit a drop in the scattering cross section. The admixture of (usually small) well-defined amounts of ortho- $\mathrm{H}_{2}$ is, therefore, an effective way of increasing the scattering cross section of the moderator medium and expected to enable a more complete thermalization. This, however, bears a trade-off, as with growing ortho- $\mathrm{H}_{2}$ content also the neutron extraction properties deteriorate to some extent.

When using optimized ortho-para mixtures instead of pure para- $\mathrm{H}_{2}$, the result could be a cryogenic moderator of an unprecedented performance, featuring the advantages of the low-dimensional design approach but mitigating the problem of an incomplete thermalization.

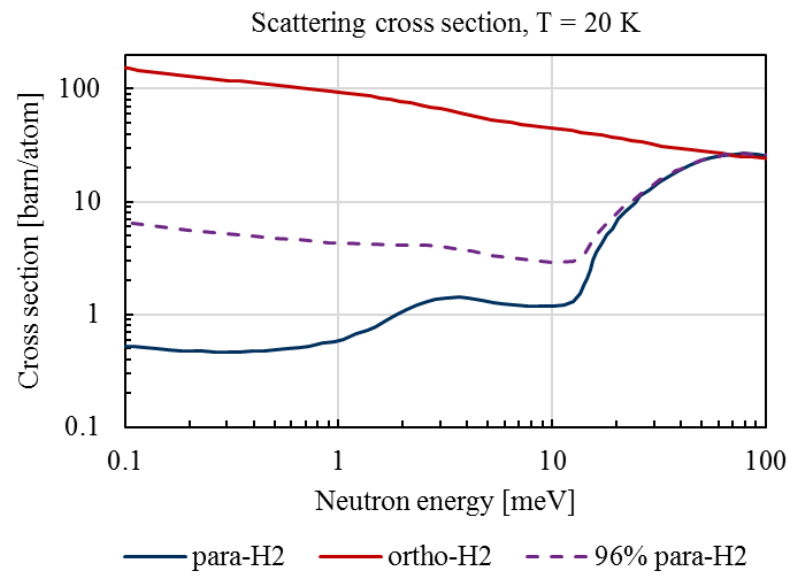

Figure 1. Scattering cross section of para- $\mathrm{H}_{2}$, ortho- $\mathrm{H}_{2}[3]$ and a mixture containing $96 \%$ para- $\mathrm{H}_{2}$ as a function of neutron energy. The data for the creation of this diagram has been extracted from plots presented in [4].

Currently, the design of cold moderators using hydrogen is subject to uncertainties due to the ambiguity in the required nuclear data [4]. Furthermore, Monte Carlo simulations of moderators using ortho-para mixtures are especially hard to manage and prone to errors $[5,6]$. To overcome this problem and enable a reliable moderator design, there are endeavors by JCNS to measure the neutron spectra resulting from different ortho-para mixtures (and pure para- $\mathrm{H}_{2}$ ) experimentally. In this work, the cryogenic system required to provide liquid hydrogen with a variable ortho-para ratio is presented.

\section{System overview}

\subsection{Cold moderator}

The design of the cold moderator vessel follows the concept of low-dimensional cold moderators as proposed by Mezei et al. [2] and was designed for pure para- $\mathrm{H}_{2}$. The MCNP-based parameter studies required for dimensioning the $\mathrm{LH}_{2}$ (liquid hydrogen) volume in the cold moderator vessel were done at the Chair of Nuclear Engineering and Technology Transfer (NET) at RWTH Aachen in cooperation with JCNS-2 $[5,6]$, whereas the final engineering design was a contribution of ZEA-1 at Forschungszentrum Jülich [7]. The result is a quasi-onedimensional cylindrical moderator vessel that is fed with thermal neutrons from all sides. Due to the high scattering cross section at thermal neutron energies (see Figure 1), most incoming neutrons are scattered down to cold neutron energies within the mean free path $1_{\text {thermal }}=1 \mathrm{~cm}$. When using pure para- $\mathrm{H}_{2}$, the scattering cross section for neutrons of energies below $15 \mathrm{meV}$ is more than one order of magnitude lower, resulting in an increased mean free path of about $1_{\text {cold }}=11 \mathrm{~cm}$ up to approx. $1.5 \mathrm{~m}$. As a consequence, the extraction of cold neutrons along the longitudinal axis of the cylindrical moderator vessel is particularly efficient, as a big moderator volume can be used to provide cold neutrons within the limited divergence required. The small diameter of the cylindrical moderator vessel allows feeding the all of its volume efficiently with thermal neutrons without the risk of depletion in the center of the moderator. The optimal moderator dimensions are a trade-off between neutron flux and brilliance at a considered sample position. The smaller the illuminated area of the cold moderator, the higher the brilliance but the smaller the flux. However, a first approximation is a diameter $d$ corresponding to $l_{\text {thermal }}$ and a length 1 corresponding to $1_{\text {cold, }}$, though for reasons of the achievable total neutron flux and manufacturing, the

dimensions $\mathrm{d}=2.5 \mathrm{~cm}$ and $1=10 \mathrm{~cm}$ have been

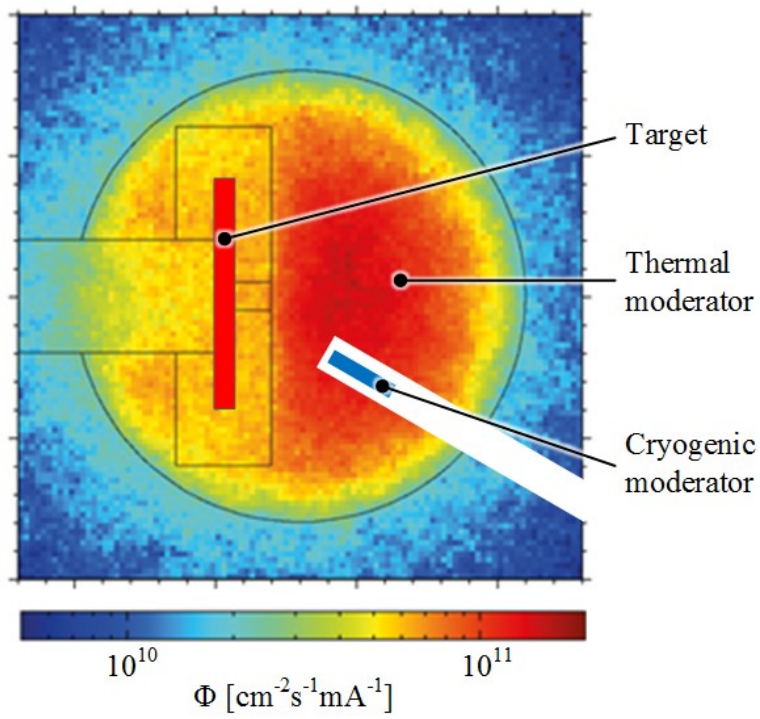

Figure 2. Schematic view of target, thermal moderator and cryogenic moderator positioned inside the thermal neutron density maximum.

chosen [6], resulting in a total liquid hydrogen volume of $200 \mathrm{ml}$. The operating temperature and absolute pressure are $\mathrm{T}=17$ to $20 \mathrm{~K}$ and $\mathrm{p}=1.3$ to $1.5 \mathrm{bar}$.

Figure 2 contains a schematic view of the potential position of the cryogenic moderator inside the extraction channel of the thermal moderator and Figure 3 shows a CAD model of the engineering design of the moderator vessel with the He cooling channels milled into its shell. The latter allow maintaining the $\mathrm{LH}_{2}$ within a narrow temperature band inside the subcooled regime. The vessel is positioned inside a long, cylindrical vacuum jacket 
which is connected to the main cryostat and can be inserted into the thermal moderator.

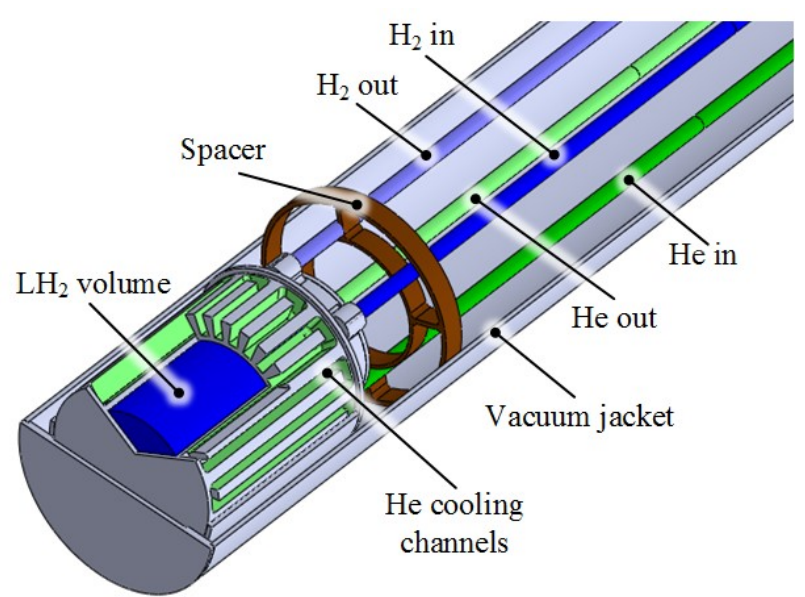

Figure 3. CAD model of the cryogenic moderator assembly.

\subsection{Process and cryostat design}

The cryogenic system is required to condense hydrogen and maintain it within the desired temperature and pressure range so that it is in a subcooled state. Filling the moderating volume shall take about $30 \mathrm{~min}$ at the design mass flow. A further central, and very challenging, requirement is the adjustability of the concentration in the range from 25 to $100 \%$ para- $\mathrm{H}_{2}$ with an accuracy of $\pm 0.1 \%$ para- $\mathrm{H}_{2}$. This represents a novelty from a cryogenics point of view - the presented cryostat is the first hydrogen liquefier with an adjustable ortho-para ratio. The concentration shall furthermore be monitored externally by a suitable measurement device. to different ortho-para ratios, but not as a final solution used for scattering experiments on a larger scale.

Figure 4 shows a schematic view of the cryogenic system. It is based on the following concept: two separate hydrogen flows, regulated by mass flow controllers (MFCs), are condensed in a counter flow heat exchanger (HX) cooled by helium cold gas. One of the hydrogen flows is converted to nearly $100 \%$ para- $\mathrm{H}_{2}$ using a ferric oxide catalyst bed contained inside the heat exchanger, whereas the other flow maintains its initial composition of $75 \%$ ortho- $\mathrm{H}_{2}$ and $25 \%$ para- $\mathrm{H}_{2}$ (also referred to as normal- $\mathrm{H}_{2}$ ). After passing the heat exchanger, the two flows are mixed together at the desired ratio, as imposed by the mass flow controllers. The mixture is fed into the moderator vessel, where it is cooled with helium cold gas passing through the cooling channels inside the outer shell.

In order to be able to control the para concentration the intended way, the ortho-para conversion in the catalyst needs to be complete and the system as a whole needs to be kept in a stable thermal equilibrium. These are significant challenges that needed to be considered during the design and commissioning process.

The moderator vessel is constantly overfilled with a defined hydrogen mass flow in order to compensate for any spontaneous para-ortho or ortho-para conversion occurring in the cryostat, to achieve reasonable mixing ratio errors associated to the mass flow controllers, and to enable the continuous monitoring of the hydrogen composition at the exhaust.

To prevent the risk of freezing hydrogen (at temperatures below $13.8 \mathrm{~K}$ ) and to stabilize the system thermally, an electrical heater is located right after the LHe inlet inside the cryostat. It evaporates and heats up the helium to about $15 \mathrm{~K}$. A second heater is located just before the triple heat exchanger $\left(\mathrm{He}\right.$, para- $\mathrm{H}_{2}$ and normal-

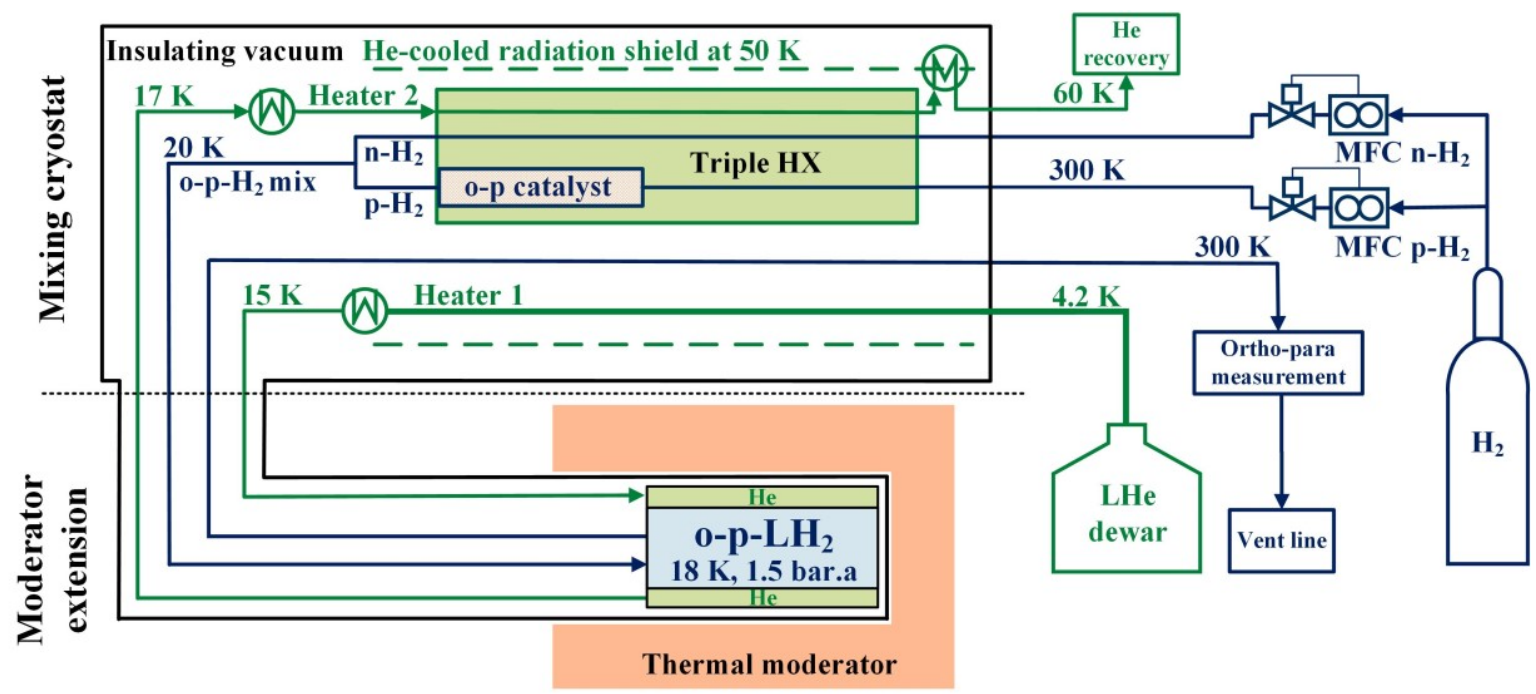

Figure 4. Schematic representation of the cryogenic system.

The process and the resulting cryostat have been designed and built over the course of the last years, mainly at TU Dresden [8]. The present system is understood as a proof-of-principle prototype for MCNP validation purposes and measurements of neutron spectra associated
$\mathrm{H}_{2}$ ), which can be used to control and stabilize the helium entry temperature at the heat exchanger independently from the helium temperature at the outlet of the moderator vessel. A helium-cooled radiation shield at about $50 \mathrm{~K}$ makes use of the remaining cooling capacity of the helium cold gas just before it leaves the cryostat. 


\section{$2.3 \mathrm{H}_{2}$ composition monitoring}

It is of crucial importance to monitor the para concentration in real time by means of an external device independent from the mixing cryostat. Even though the mix composition, in theory, can be predicted quite easily knowing the separate para- $\mathrm{H}_{2}$ and normal- $\mathrm{H}_{2}$ mass flows and their corresponding para concentrations, transient thermal states in the cryostat, as well as undesired orthopara (or para-ortho) conversion, can potentially cause a mix concentration that differs from the prediction.

Currently, a speed of sound-based approach is being pursued at TU Dresden. Good experiences have been made with the binary gas analyzer BGA244HP by Stanford Research Systems, which allows an uncomplicated online monitoring of the speed of sound in gaseous phase. The para concentration is obtained from linear interpolation between two reference points: normal$\mathrm{H}_{2}$ (25\% para- $\mathrm{H}_{2}$, directly from the bottle) and hydrogen with the equilibrium composition corresponding to $77.3 \mathrm{~K}\left(51 \%\right.$ para $-\mathrm{H}_{2}$, produced in an oversized reference catalyst immersed in a liquid nitrogen bath).

Because ortho- and para- $\mathrm{H}_{2}$ differ only very slightly in most physical properties, measuring the para concentration is a rather challenging task. Due to the tough accuracy requirement, the concentration measurement is repeatedly calibrated by feeding it alternately with either one of the two reference gases over the course of an experiment. This accounts for small drifts in the measured speed of sound that can occur over the course of several hours. Furthermore, great efforts are made to keep any contaminants in the analysed hydrogen gas to a minimum, as they can significantly affect the measurement accuracy. The device and all pipes are extensively pumped and purged with pure hydrogen and the residual water content of the gas leaving the speed of sound system is continuously monitored using a dew point transmitter.

Determining a definite value for the absolute error in terms of para concentration is an intricate and ongoing task. However, a significant amount of experience using the device for measuring concentrations between $25 \%$ and $51 \%$ para- $\mathrm{H}_{2}$ has been gathered, indicating a repeatability of $< \pm 0.3 \%$ para $-\mathrm{H}_{2}$ when used in the above described way. At concentrations higher than $51 \%$, accuracy and repeatability of the measurements are expected to deteriorate with an increasing para concentration if no third reference gas with $\approx 100 \%$ para- $\mathrm{H}_{2}$ can be used for calibration purposes. At the moment, there is still no independent device for the production of nearly pure para- $\mathrm{H}_{2}$ existing at the lab.

A promising way to increase the sensitivity of such speed of sound-based concentration measurement is reducing the gas temperature. The difference between the sound speeds of ortho- $\mathrm{H}_{2}$ and para- $\mathrm{H}_{2}$ spreads up at lower temperatures until it reaches a maximum at around $130 \mathrm{~K}$ [8] (see Figure 5). For this reason, a measurement rack that allows an active cooling of the device down to temperatures around $230 \mathrm{~K}$ is being developed, promising three- to four-fold sensitivity in terms of para concentration compared to the current setup at room temperature.

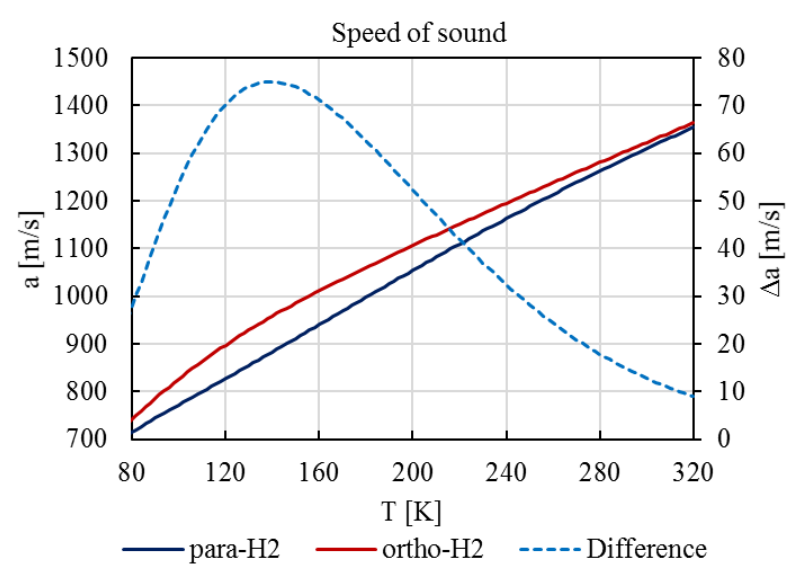

Figure 5. Speed of sound a of ortho- $\mathrm{H}_{2}$ and para- $\mathrm{H}_{2}$ and their difference $\Delta \mathrm{a}$ vs. temperature $\mathrm{T}$.

\section{Commissioning}

\subsection{General status}

First test runs of the system were done already in August 2017 at AKR-2 [8] (a zero-power training reactor located at the campus of TU Dresden). However, several problems caused an unreliable operation of the system, most importantly insufficient vacuum pressure and cold leaks in the process lines. Over the course of the last year, the system has been reworked extensively to ensure leak tightness and a good insulating vacuum. Furthermore, the cryostats periphery has been improved and controls and data acquisition methods have been revised entirely.

Now, the system can in principle be operated in the intended way. For the first time, test runs have been made where a steady state could be maintained for several hours. A cool-down from room temperature takes about 3 hours, followed by 1 to 2 hours required to establish a stable operating point producing $\mathrm{LH}_{2}$ at the desired pressure, temperature and para concentration. When maintained cool overnight by using a small LHe flow, reestablishing operating temperature is a matter of about 20 min the next day.

Commissioning is still ongoing, but great progress has been made during the last months. It could be shown that the cryostat works as designed.

\subsection{Demonstration of mixing operation}

Several tests have been run in order to demonstrate that the mixing cryostat is capable of producing different mix compositions. For reasons of safety, reduced complexity and experimentation time, these tests were done using short circuits instead of connecting the moderator vessel to the mixing cryostat. The cryostat was cooled down, brought into a stable thermal state, and the He temperature was reduced such that hydrogen condensation could take place inside the heat exchanger. Afterwards the setpoints of the two hydrogen MFCs were adjusted in such way that several different para concentrations could be produced 
subsequently, in the here presented test from $60 \%$ to $100 \%$ in steps of $10 \%$.

To allow a sensible prediction of the mix concentration by using the mass flows controlled via the MFCs, it is a prerequisite to know the para concentrations of the two hydrogen flows. In particular, this means that the ortho-para conversion towards the temperaturedependent equilibrium state in the ferric oxide catalyst needs to be complete. Prior tests on the catalyst performance have shown that full conversion takes place in all relevant operating points (i.e. combinations of $\mathrm{He}$ and $\mathrm{H}_{2}$ mass flows and He heater temperature setpoints).

Figure 6 contains measurement data taken during one of the mixing tests, showing the predicted and measured mix concentration over the course of almost three hours.

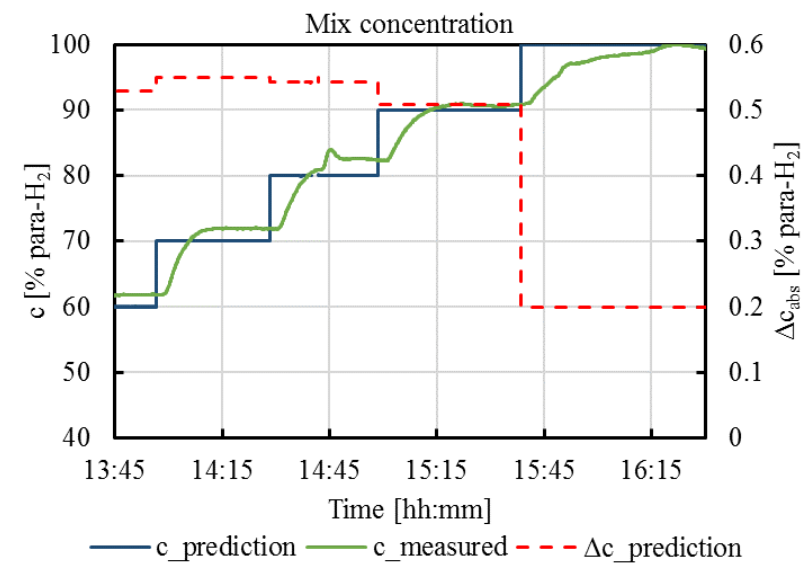

Figure 6. Para concentration during mixing test. The prediction is based on the measured partial mass flows and assumed concentrations of $99.9 \%$ para- $\mathrm{H}_{2}$ and $25.0 \%$ para- $\mathrm{H}_{2}$ respectively. The absolute prediction error is based on errors in the partial mass flows and an assumed absolute error of $0.2 \%$ para- $\mathrm{H}_{2}$ after catalyst. The measured values are based on the speed of sound measurement calibrated using normal- $\mathrm{H}_{2}$ and para- $\mathrm{H}_{2}$ as reference gases.

The prediction is based on measurements of the hydrogen mass flows and assumed para concentrations (99.9\% and $25.0 \%$, respectively) of the two partial flows. The measured concentrations are based on the speed of sound readings.

It could be shown that the mix concentration can be controlled by the user as intended. The hydrogen MFCs respond very quickly and operate in an extremely stable way, which explains the perfectly step-like shape concentration prediction in Figure 6 . When the mixing ratio of the partial streams is changed to adjust the orthopara ratio, the measured concentration lags behind the predicted one, exhibiting a settling time of about $30 \mathrm{~min}$ using $30 \%$ of the maximum hydrogen flow. On the one hand, this is because the hydrogen inventory in the cryostat needs to be replaced entirely. Especially volumes of condensed hydrogen, e.g. contained in the process pipes after the mixing location, cause a long response time. On the other hand, changing the partial hydrogen mass flows imposes a disturbance on the system, causing a thermal transient in the heat exchanger and catalyst bed, which in turn influences the mix composition. To promote a high mixing accuracy during nominal operation as neutron moderator, the system should be run in only one operating point after $\mathrm{H}_{2}$ condensation has started, and emptied entirely from condensate before a different operating point is targeted.

After the system had settled in each operating point, prediction and measurement coincided within about $+2.7 \%$ para- $\mathrm{H}_{2}$.

This is still far from the desired accuracy of $\pm 0.1 \%$. However, the goal of the present test was not to achieve the highest possible mix composition accuracy, but rather to demonstrate mixing operation for the first time and examine the systems response to changes in the concentration setpoint.

One of the reasons for the notable deviation between measurement and prediction is probably a poor measurement accuracy during the test. In order to allow a continuous tracking of the concentration without interruptions, no recalibration of the concentration measurement was done over the course of the experiment. There was only one reference measurement with normal$\mathrm{H}_{2}$ before the test, and one with nearly pure para- $\mathrm{H}_{2}$ afterwards, with a time span of several hours in between. Usually, reference measurements should be done every 30 min to account for drifts in the speed of sound device. Currently, the only way of obtaining pure para- $\mathrm{H}_{2}$ as a reference gas for calibration is producing it in the cryostat itself. Because this would mean an interruption in the operation at the desired mix composition and impose a large disturbance on the system, reference measurements like this can, at the most, be done in the very beginning or end of an experiment.

However, it could be shown that the mixing cryostat works as intended and it is ready for use with the moderator vessel.

\section{Conclusion}

In the frame of the HBS project, a holistic optimization of the chain from neutron production to neutron detection is a key objective, and the performance of the cryogenic moderator plays a vital role. In a joint project, FZ Jülich and TU Dresden are developing an experimental setup to test a low-dimensional liquid hydrogen moderator with a variable ortho-para ratio for the first time. Usually, pure para- $\mathrm{H}_{2}$ is used for cryogenic moderators. However, by adding small, well-defined amounts of ortho- $\mathrm{H}_{2}$, the neutron spectrum can potentially be shifted towards lower energies while maintaining the good neutron extraction properties that the low-dimensional (para- $\mathrm{H}_{2}$ ) moderator approach allows. In this work, the cryogenic system for the provision of $\mathrm{LH}_{2}$ with adjustable ortho-para ratio and its metrological monitoring via speed of sound measurements are presented. The commissioning of the system has advanced to a point where it is ready to be used with the cryogenic moderator vessel. It could be shown that the main cryostat can produce liquid hydrogen with an arbitrary para concentration between $25 \%$ and nearly $100 \%$ para- $\mathrm{H}_{2}$.

However, several steps have to follow to complete the commissioning. As a next step, the system needs to be tested with the moderator vessel, and investigations 
regarding the achievable mixing accuracy have to be conducted. Furthermore, the speed of sound-based concentration measurement will be refined. Afterwards, first tests under neutron radiation are to follow soon.

\section{References}

1. U. Rücker, T. Cronert, J. Voigt, J.P. Dabruck, P.E. Doege, J. Ulrich, R. Nabbi, Y. Beßler, M. Butzek, M. Büscher, C. Lange, M. Klaus, T. Gutberlet, T. Brückel, Eur. Phys. J. Plus 131, 19 (2016)

2. F. Mezei, L. Zanini, A. Takibyev, K. Batkov, E. Klinkby, E. Pitcher, T. Schönfeldt, J. Neutron Res. 17, pp.101-105 (2014)

3. M. B. Chadwick et al., Nucl. Data Sheets 112, pp. 2887-2996 (2011)

4. K. B. Grammer et al., Phys. Rev. B 91, 180301(R) (2015)

5. J. P. Dabruck: Simulation Studies on the Target Station for the High-Brilliance Neutron Source $H B S$, PhD Thesis, NET - RWTH Aachen (2018)

6. T. Cronert, J. P. Dabruck, P.-E. Doege, Y. Beßler, M. Klaus, P. Zakalek, U. Rücker, C. Lange, M. Butzek, W. Hansen, R. Nabbi, T. Brückel, J. Phys.: Conf. Ser. 746, 012036 (2016)

7. M. Hofmann: Auslegung eines kryogenen Flussfingers im Rahmen einer Parameterstudie am AKR-2 als Voruntersuchung zur Entwicklung eines hoch-brillianten Neutronenquelle, BSc Thesis, FH Aachen (2016)

8. M. Klaus, A. Schwab, Ch. Haberstroh, E. Eckhardt, Y. Beßler, J. Baggemann, T. Cronert, IOP Conf. Ser.: Mater. Sci. Eng. 502, 012161 (2019) 